\title{
The approach of creating a particular postprocessor and using CNC measuring cycles
}

\author{
Georgi M. Martinov ${ }^{1}$, Anna V. Stas ${ }^{1, *}$, and Oleg A. Kudinov ${ }^{1}$ \\ ${ }^{1}$ FSBEI HPE MSTU “STANKIN”, Moscow, Russia
}

\begin{abstract}
The article is devoted to research, development and testing of the postprocessor for the $\mathrm{CNC}$ system. In the article was made a comparison between the methods for creating postprocessors, was described the development of a postprocessor and of the cycle of determining the diameter of the shaft and the coordinates of its center, used in testing postprocessor. In the work are demonstrated the tests of the developed postprocessor. As a result, after the work of the postprocessor, there is a finished $\mathrm{NC}$ for the $\mathrm{CNC}$ system.
\end{abstract}

In industrial enterprises the majority of $\mathrm{CNC}$ equipment is used. Such as metal-cutting machines (milling, turning, machining centers), grinding, plasma, laser machines. There is a wide variety of manufacturers of $\mathrm{CNC}$ systems and each tries, to add new solutions to the standard ISO code that would distinguish it from competitors. Programs written using Gcode have a rigid structure. Commands are combined into frames - groups of one or more commands, their sequence in the frame can be arbitrary, but usually it is strictly regulated.

This standard, despite the years of the past, is supported by all modern manufacturers of $\mathrm{CNC}$ systems, it is necessary for ease of transfer of control programs from one system to another. Nevertheless, many manufacturers introduce their own standards, which work only on their products. This is justified by the fact that they want to make the programming of the system easier and easier for ordinary users. In some systems, these differences are almost not noticeable, while in others they completely change the programming technique. This is realized either by changing the functionality of standard $\mathrm{G}$ or $\mathrm{M}$ codes or by implementing its own functions, which are not available from other manufacturers. Figure 1 shows a diagram of the necessary diversity of postprocessors in enterprises.

The variety of manufacturers of CNC systems in industrial plants leads to the fact that when programming equipment using CAM-systems it is necessary to have a separate postprocessor for each CNC system. To reduce the number of postprocessors for machines with different $\mathrm{CNC}$ systems used within a single enterprise or a mechanical workshop, it is proposed to develop a method for generating a $\mathrm{CP}$, in which the postprocessor, by inputting data on the CNC system, independently made corrections to the base code of the $\mathrm{CP}$, based on its syntax. [1]

\footnotetext{
*Corresponding author: anna.stas2015@yandex.ru
} 


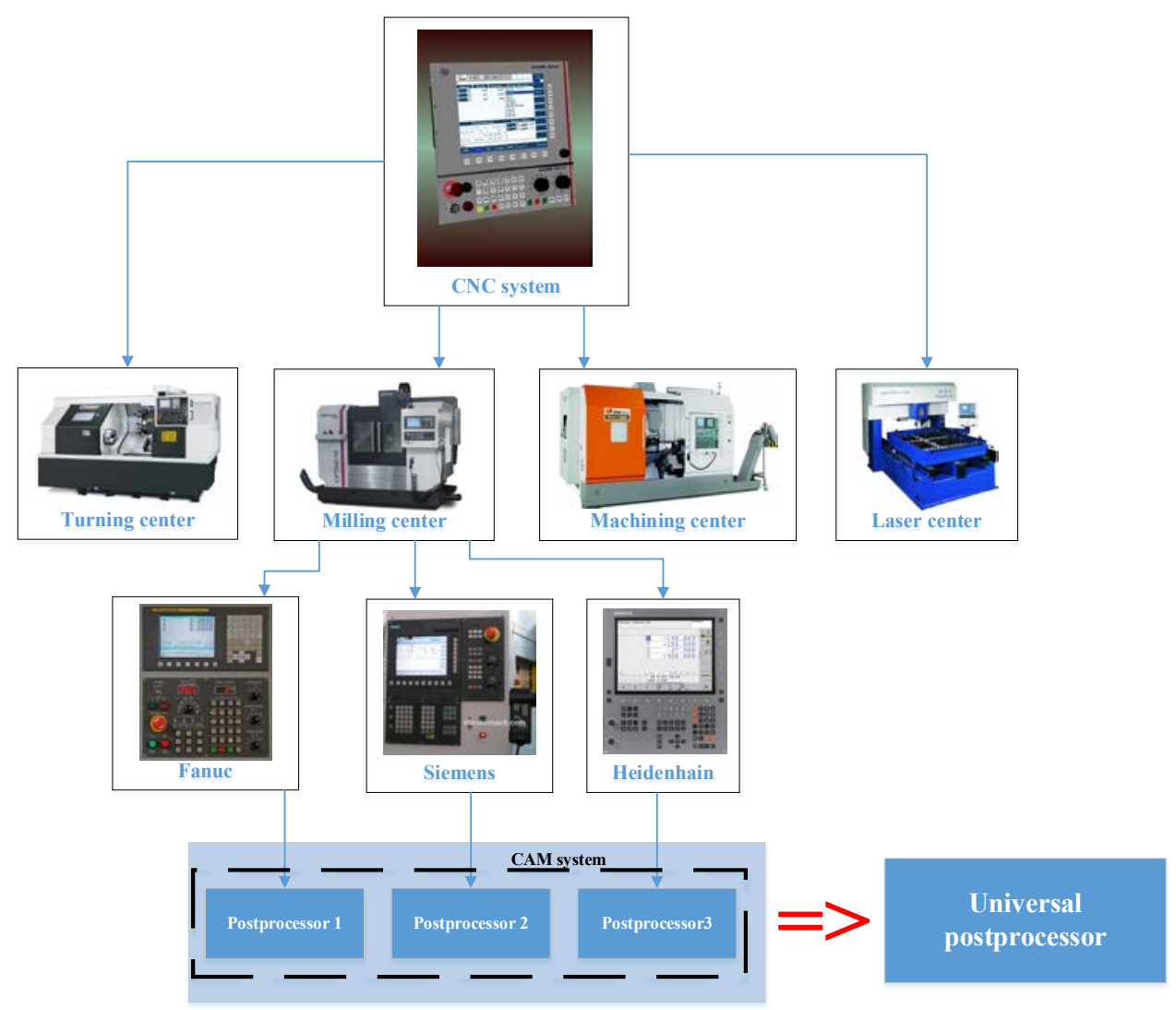

Fig. 1. Schematic representation of the diversity of postprocessors for machines with CNC systems.

Comparison of methods for creating postprocessors produces in the article "Ideology of postprocessing in modern CAD / CAM-systems" Filipovich K.V: The individual method is that a postprocessor is created for each individual machine in a high-level language, for example, $\mathrm{C}++$; generalized method is similar to the previous one by the development method, but used for a group of similar equipment; the universal metod is that special generators are used to create the postprocessor, in which it is necessary to enter all the data about the machine and the $\mathrm{CNC}$ system; invariant method is creating a huge base with the existing machines and $\mathrm{CNC}$ systems, its main drawback is the need for constant updating. All methods have their own advantages and disadvantages.[2]

The development of the method will be based on the universal method of postprocessor development, which will be implemented in the built-in generator NC-GPOST CAD Creo Parametric. This editor allows using a flexible approach to creating postprocessors due to the FIL language, which includes various constructs such as cycles, conditions, switch-case operators, postprocessor for the Fanuc CNC system. Figure 2 shows the scheme for implementing the approach. 


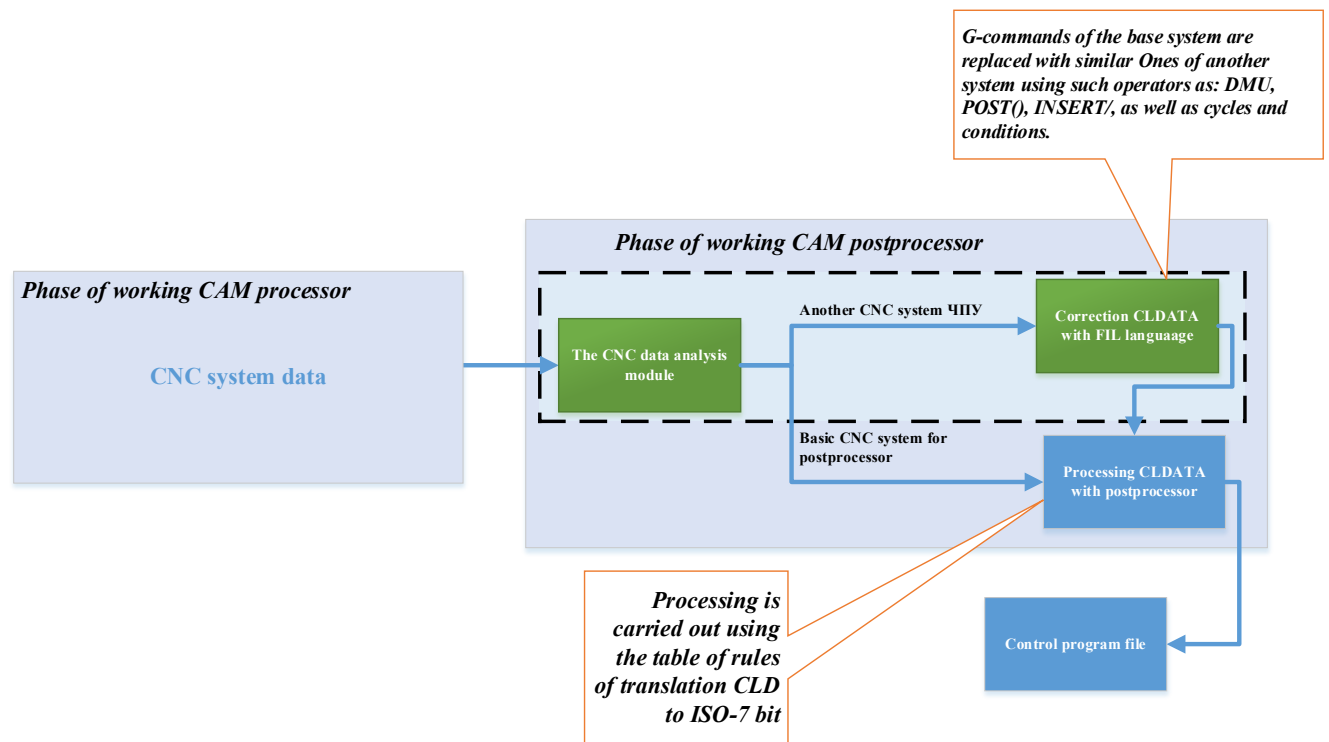

Fig. 2. The postprocessing phase.

At the stage of work of the postprocessor phase, processing of the data about the movement of the tool and various technological commands takes place. The data is transferred from the CLData format accepted in the CAM system to a specific CNC system, for example, Axiom Control code ISO-7 bit. Data is translated from one form to another based on a special correspondence table that contains rules for translating the syntax.

Table 1. Fragment of the translation rules table for the CLDATA language in ISO-7 bit

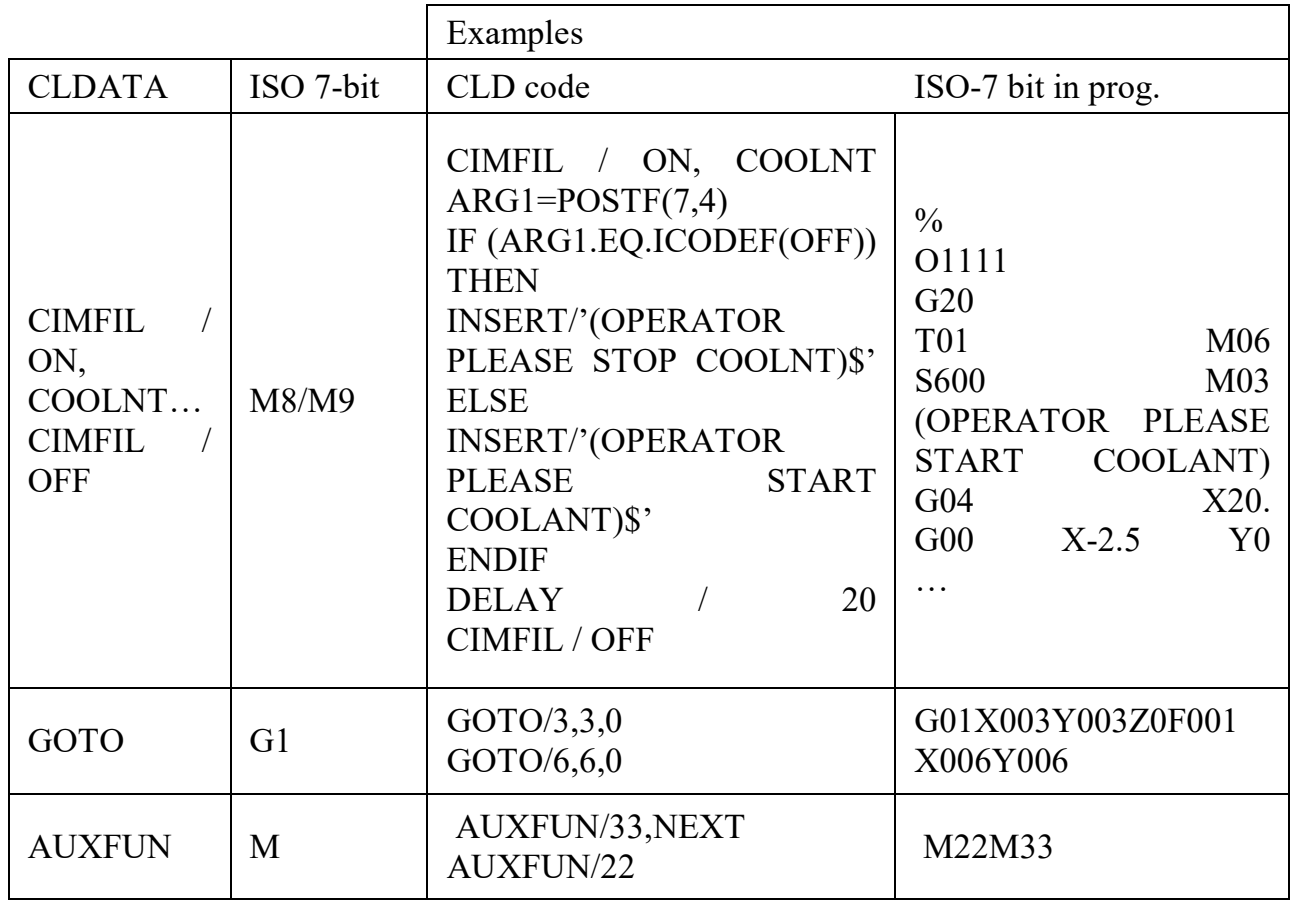


In the approach the algorithm is built into the postprocessor already ready for a particular CNC system, which will translate the CLD file into code, an alternative to the CNC system

in our case, Axioma Control. This module is implemented in the CAD Creo Parametric, because it has the necessary tools for this, which allows you to make corrections to the existing postprocessor using a special tool (FIL Editor). This system allows you to design postprocessors for machines of different types, starting with lathes and ending with presses and laser machines. It is worth noting the editing unit of the FIL file, with which the user can add certain commands to the postprocessor by manually programming the CLDATA data. If necessary, using this block, you can make a more flexible setting of the postprocessor. [3]

To conduct tests on the basis of the finished 3D model, data on the workpiece and parameters of the cutting tool in the CAM-module Creo Parametric, the tool path was developed to obtain the required geometric shape of the workpiece. Before starting the workpiece processing cycle, it is necessary to enter its geometric parameters into the CNC system, and also to combine the machine coordinate system with the program coordinate system. This is necessary for the correct operation of the control program. The measurement data can be carried out using the MeasuringHall () cycle of measuring the diameter of the workpiece and determining its center in case the control program is calculated from the center of the model. The line for calling the loop should also be placed in the Fil-file obtained by the postprocessor method being developed for the control system Axioma Control.

The purpose of the cycle is to determine the diameter and center of the shaft. The cycle of measuring of shaft is conducted in 2 steps:

-measurement of the shaft at 4 points parallel to the axes;

-calculation of the diameter and position of the shaft center.

The cycle of measuring of shaft is using the next input and output parameters (Table 1)

Table 2. Input and output parameters for the cycle of measuring of shaft

\begin{tabular}{|c|c|c|}
\hline № & Parameter & Description of the parameter \\
\hline \multicolumn{3}{|c|}{ Input parameters } \\
\hline 1. & Radius & Preliminary radius of shaft \\
\hline 2. & Plane & Working plane (XY (G17), XZ (G18), YZ (G19)) \\
\hline 3. & Tool number & Touch probe's position \\
\hline 4. & Safe height & Safe height above the workpiece \\
\hline 5. & Safe distance & $\begin{array}{l}\text { Distance between the ball of the probe and the } \\
\text { workpiece }\end{array}$ \\
\hline 6. & Feed rate & Measurement speed \\
\hline 7. & Depth of measurement & Distance of measurement \\
\hline 8. & Overrun & Overrun \\
\hline \multicolumn{3}{|c|}{ Output parameters } \\
\hline 1. & Diameter & $\begin{array}{l}\text { The actual diameter relative to the } 1 \text { and } 2 \text { axes of the } \\
\text { working plane }\end{array}$ \\
\hline 2. & Diameter difference & $\begin{array}{l}\text { The difference of diameter relative to the } 1 \text { and } 2 \text { axes } \\
\text { of the working plane }\end{array}$ \\
\hline 3. & $\begin{array}{l}\text { Coordinates of the } \\
\text { center }\end{array}$ & Coordinates of the shaft center in 3 axes $(\mathrm{X}, \mathrm{Y}, \mathrm{Z})$ \\
\hline 4. & $\begin{array}{l}\text { Difference of oordinates } \\
\text { of the center }\end{array}$ & $\begin{array}{l}\text { The difference of coordinates of the shaft center } \\
\text { relative to } 3 \text { axes }(X, Y, Z)\end{array}$ \\
\hline
\end{tabular}


The article considers the variant of measurement in the plane XY. Before starting the shaft measurement cycle, the operator enters the values of the input parameters. Next, the probe is moved to the 1 st point with feed rate in the positive direction relative to the OX axis to the safe distance (point 1.1.). Next, with the calibration rate, the probe moves to the point of the deviation of the probe (point 1). If there is no signal about the deflection of the probe, the movement continues to the distance of the overrun (point 1.2). If the probe was deviationed, the coordinates of the point are stored in the NC memory, otherwise an error message is given and the cycle is interrupted. Then the second point is measured (respectively, points $2.1,2,2.2$ ), in the negative direction relative to the OX axis. The coordinates of the 2 nd point are stored in the CNC memory, the real center of the shaft is calculated relative to the $\mathrm{OY}$ axis and the diameter along the $\mathrm{OX}$ axis. The probe moves to the calculated center (point 0 ). Then from the calculated center, the probe measures points 3 and 4 in the positive and negative direction along the OY axis, CNC calculates the shaft center relative to the $\mathrm{OX}$ axis and the shaft diameter along the $\mathrm{OY}$ axis.

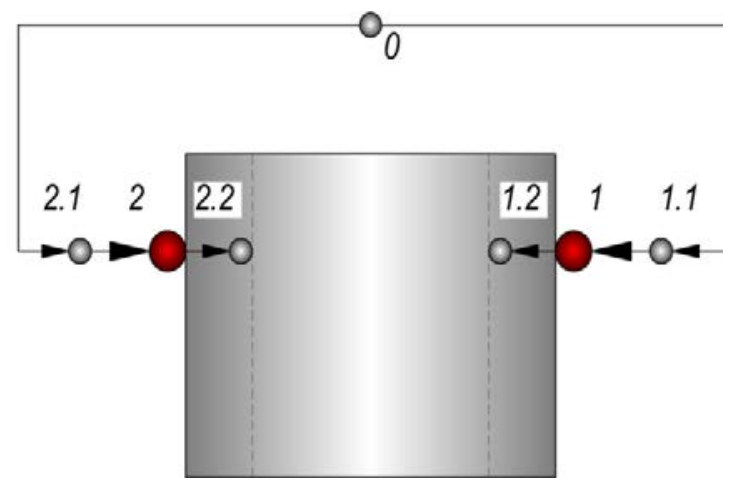

Fig. 3. The principle of the shaft measurment.

Then, the coordinates of the shaft center are entered in the table of offset of the 0 point of workpiece, which is located in a special window of the CNC system. [4]

Data on the tool path and the processing parameters of the processing are contained in the CLDATA file, which is not understandable to any CNC system. In order to get the control program based on it, you need to use a special file - a postprocessor, which allows you to translate data from the CLD format into code that is understandable for the CNC system. For this case, a postprocessor developed according to the described method and adapted for the control system Axioma Control was used. 


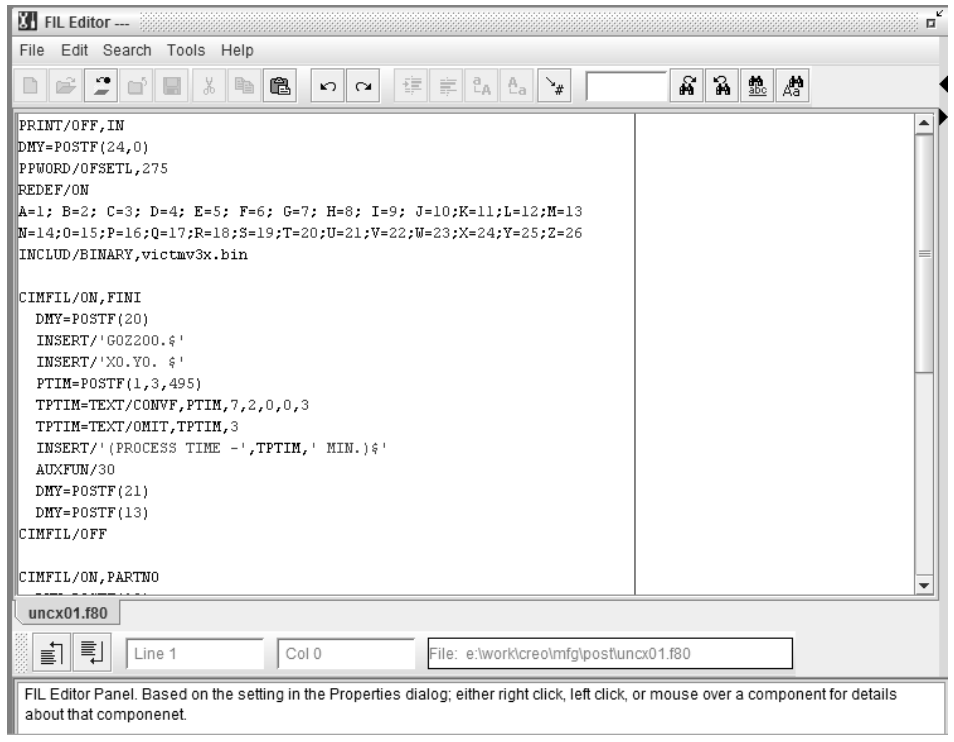

Fig. 4. The program for generation of postprocessors NC-GPOST.

As a result, after the work of the postprocessor, there is a ready-made control program for the CNC control system Axiom Control, which initially measures the workpiece, storing data into the system memory, and then machining the part to achieve the specified geometric shape. Fragment of the program:

G00 G15 G17 G40 G49 G53 G71 G80 G90 G94 G97 G153 G191 G193 // Security string MeasureHall (50, G17, 75, 5, 5, 500, 5, 5, G54, P2) // call the shaft measuring cycle N10 G154 P2// Calling the Offset Function .

\section{$\mathrm{S} 4500 \mathrm{M} 3$}

G0X52.052Y-70.012

G1X-51.456

The part obtained after the program operation is shown in Figure 5.

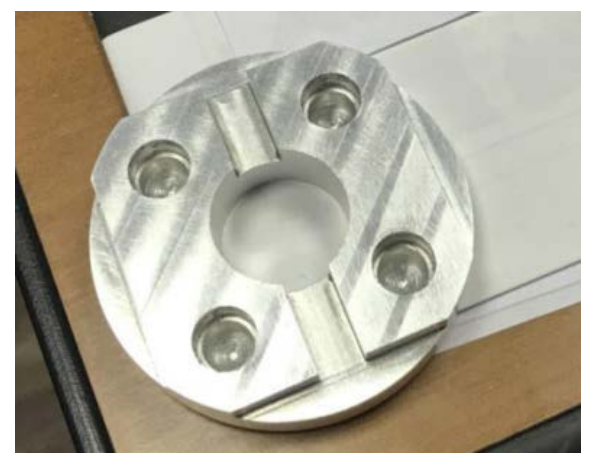

Fig. 5. Machined part.

This method allows you to significantly reduce the time of writing control programs and their transfer from one equipment to another by reducing the labor costs for designing a new postprocessor. Instead, it is necessary to adapt the existing postprocessor to the new $\mathrm{CNC}$ system by making corrections in the FIL language. With the further development of the method, it is also possible to adapt several systems at the same time based on one basic postprocessor. 


\section{References}

1. S.N. Grigoriev, A.A. Kutin, V.A. Dolgov, Principles of construction of digital production in mechanical engineering, Vestnik MGTU Stankin, No. 4 (31), P. 10-15 (2014)

2. M.I. Barancekova, A. A. Gusev, B. U. Kramarenko. The design of the technology. The textbook for the engineering students Under the General editorship of Y. M. Solomentsev (Moscow: Mashinostroenie, 1990, p. 354-359)

3. O. A. Kudinovon, Approach to developing post processors for CNC machines milling group. Computer integration of production and IPI-technologies: proceedings of VIII all-Russian scientific-practical conference. - Orenburg, 2017. - 704 p. ISBN 978-57410-1850-7. p. 87-91

4. L. I. Martynova, A. V. Stas, The Research and development of automatic cycle controlling the position of workpieces on the CNC machines. Proceedings of the XVII international scientific and practical conference " systems of design, technological preparation of production and management stages of the life cycle of an industrial product (CAD/CAM/PDM - 2017). Under the General editorship of A. V. Tolok. In-t problem UPR. they. V. A. Trapeznikova.-Electron. text Dan. - Moscow: IPU ran, 2017. C. $63-67$ 